\title{
AROUND THE RELATIVE CENTER PROPERTY IN ORTHOMODULAR LATTICES
}

\author{
G. CHEVALIER
}

(Communicated by Louis J. Ratliff, Jr.)

\begin{abstract}
The paper deals with the relative center property in orthomodular lattices (OMLs). The property holds in a large class of OMLs, including locally modular OMLs and projection lattices of $\mathrm{AW}^{*}$ - and $\mathrm{W}^{*}$-algebras, and it means that the center of any interval $[0, a]$ is the set $\{a \wedge c \mid c$ central in $L\}$. In $\S 1$ we study the congruence lattice of an OML satisfying the Axiom of Comparability (A.C.) and, in $\S 2$, we prove that the central cover of an element can be expressed in many different ways in OMLs satisfying a certain condition (C). For complete OMLs, Axiom (A.C.) and condition (C) are equivalent to the relative center property. In $\S 3$, we give a coordinatization theorem for complete OMLs with the relative center property.
\end{abstract}

\section{INTRODUCTION}

This paper is composed of three sections, all dealing with the relative center property in orthomodular lattices (abbreviated OMLs). This property first appeared in the work of $J$. von Neumann on continuous geometries; it holds in an OML $L$ if the center of any interval $[0, a]$ of $L$ is the set $\{a \wedge c \mid c$ central in $L\}$. The class of OMLs satisfying the relative center property is large; it contains locally modular OMLs, lattices of projections of Baer ${ }^{*}$-rings and various types of operator algebras. One aim of this paper is to demonstrate the importance of this property in the theory of OMLs.

In $\S 1$, we consider the class of OMLs satisfying a property called the Axiom of Comparability and denoted (A.C.). Different equivalent forms of (A.C.) are given, and we prove interesting results concerning the congruence lattice of an OML with (A.C.). This section begins by recalling that for complete OMLs, (A.C.) is equivalent to the relative center property.

In $\S 2$, we consider OMLs satisfying a condition noted (C). In such OMLs the central cover of an element can be expressed many different ways and some applications to Baer ${ }^{*}$-rings and operator algebras are given. We end this section by proving that every complete OML with the relative center property satisfies (C).

Received by the editors February 23, 1990 and, in revised form, June 4, 1990.

1980 Mathematics Subject Classification (1985 Revision). Primary 06C15; Secondary 20M99, $46 \mathrm{~K} 99$. 
Section 3 is devoted to the question, Why does an OML which is the lattice of projections of a Baer " -ring have the relative center property? The answer is given by a coordinatization theorem for OMLs with the relative center property; roughly speaking, it is as follows: In a Baer ${ }^{*}$-ring, a projection which commutes with every projection commutes with every element.

Notations and definitions are taken from [13], with some exceptions: kernels of congruences in OMLs are called orthomodular ideals, $\sim$ denotes strong perspectivity, and $|x|$ is the central cover of $x$. For $\S 2$, a good knowledge of [12] or $\S 8$ of [13] is useful; information about Baer ${ }^{*}$-semigroups are in [9] and [13]; and, for Rickart and Baer "-rings, [2] is the basic book.

\section{THE AXIOM OF COMPARABILITY}

1.1. Generalities. Recall a result of [8] in which $x \leqslant y$ means that there exists $z$ such that $x \sim z \leq y$ :

Proposition 1. In a complete orthomodular lattice $L$ the following statements are equivalent:

(a) $L$ has the relative center property.

(b) For all $x, y \in L$, there exists a central element $h$ such that:

$$
x \wedge h \lesssim y \wedge h \text { and } y \wedge h^{\perp} \lesssim x \wedge h^{\perp} .
$$

Actually, statement (b) implies the relative center property in any OML. If $c$ is a central element of an interval $[0, a]$ of an OML then, by statement (b) applied to $c$ and $c^{\perp} \wedge a$, there exists a central element $h$ such that $c \wedge h \lesssim$ $c^{\perp} \wedge a \wedge h$ and $c^{\perp} \wedge a \wedge h^{\perp} \lesssim c \wedge h^{\perp}$; and, as $c$ and $c^{\perp} \wedge a$ are central in [0,a], we have $c \wedge h \leq c^{\perp} \wedge a \wedge h$ and $c^{\perp} \wedge a \wedge h^{\perp} \leq c \wedge a$. Hence $c \wedge h=c^{\perp} \wedge a \wedge h^{\perp}=0$ and, finally, $c=c \vee\left(c^{\perp} \wedge a \wedge h^{\perp}\right)=a \wedge h^{\perp}$. Statement (b) is also useful for proving interesting results about the congruence lattice of a general OML (see the second part of this section) and this motivates the introduction of the following definition.

Definition 1. An orthomodular lattice $L$ is said to satisfy the Axiom of Comparability (abbreviated A.C.) if it satisfies condition (b) of Proposition 1.

We first prove some equivalent forms of axiom (A.C.). Results and proofs are similar to those obtained in Baer ${ }^{*}$-rings where equivalence of projections replaces strong perspectivity (see $[2, \S 14])$. Nevertheless, proofs are necessary for two reasons:

The theorem of additivity of strong perspectivity in OMLs [13, §7] has stronger hypotheses than the corresponding result about equivalence of projections.

In every OML, $\varphi_{a}(b)=a \wedge\left(a^{\perp} \vee b\right)$ is strongly perspective to $\varphi_{b}(a)$ and the corresponding property for equivalence of projections, the so-called parallelogram law, is not satisfied by all Baer ${ }^{*}$-rings [2, $\S 13$, Exercise 1]. 
Two elements $a$ and $b$ of an OML are said to be strongly orthogonal, written $a \Perp b$, if there exists a central element $h$ such that $a \leq h$ and $b \leq h^{\perp}$.

Proposition 2. The following statements on an orthomodular lattice $L$ are equivalent:

(a) L satisfies (A.C.).

(b) For all $a, b \in L$, there exist orthogonal decompositions:

$$
a=a^{\prime} \vee a^{\prime \prime}, b=b^{\prime} \vee b^{\prime \prime} \quad \text { with } a^{\prime} \backsim b^{\prime} \text { and } a^{\prime \prime} \Perp b^{\prime \prime} .
$$

Proof. Assume that $L$ satisfies (A.C.) and let $a, b \in L$. There exist $c, d \in L$ and $h \in \mathrm{C}(L)$ such that

$$
a \wedge h \backsim c \leq b \wedge h \text { and } b \wedge h^{\perp} \backsim d \leq a \wedge h^{\perp} .
$$

Consider: $a^{\prime}=(a \wedge h) \vee d, a^{\prime \prime}=a \wedge a^{\perp}, b^{\prime}=\left(b \wedge h^{\perp}\right) \vee c, b^{\prime \prime}=b \wedge b^{\prime \perp}$. Clearly, $a=a^{\prime} \vee a^{\prime \prime}, b=b^{\prime} \vee b^{\prime \prime}, a^{\prime} \perp a^{\prime \prime}$, and $b^{\prime} \perp b^{\prime \prime}$ hold. We have:

$$
a^{\prime \prime}=a \wedge\left[\left(a^{\perp} \vee h^{\perp}\right) \wedge d^{\perp}\right]=a \wedge h^{\perp} \wedge d^{\perp} \leq h^{\perp}
$$

and

$$
b^{\prime \prime}=b \wedge\left[\left(b^{\perp} \vee h\right) \wedge c^{\perp}\right]=b \wedge h \wedge c^{\perp} \leq h
$$

and hence $a^{\prime \prime} \Perp b^{\prime \prime}$ holds. Since $(a \wedge h) \vee c \perp\left(b \wedge h^{\perp}\right) \vee d$, we can apply the additivity theorem for strong perspectivity and $(a \wedge h) \vee d \backsim\left(b \wedge h^{\perp}\right) \vee c$, that is, $a^{\prime} \backsim b^{\prime}$.

Now assume that $L$ satisfies condition (b) and let $a, b \in L$. There exist orthogonal decompositions of $a$ and $b$ such that

$$
a=a^{\prime} \vee a^{\prime \prime}, b=b^{\prime} \vee b^{\prime \prime}, a^{\prime} \backsim b^{\prime}, a^{\prime \prime} \leq h \text {, and } b^{\prime \prime} \leq h^{\perp} \quad \text { with } h \in \mathrm{C}(L) \text {. }
$$

We have:

$$
\begin{gathered}
a \wedge h^{\perp}=\left(a^{\prime} \wedge h^{\perp}\right) \vee\left(a^{\prime \prime} \wedge h^{\perp}\right)=a^{\prime} \wedge h^{\perp} \backsim b^{\prime} \wedge h^{\perp} \leq b \wedge h^{\perp}, \\
b \wedge h=\left(b^{\prime} \wedge h\right) \vee\left(b^{\prime \prime} \wedge h\right)=b^{\prime} \wedge h \backsim a^{\prime} \wedge h \leq a \wedge h,
\end{gathered}
$$

and thus (A.C.) holds.

Proposition 3. Let $x$ and $y$ be two elements of an orthomodular lattice $L$ satisfying (A.C.). There exists a central element $h$ such that:
$(\text { A.C. })^{\prime}$

$$
x \wedge h \lesssim y \wedge h \text { and } x^{\perp} \wedge h^{\perp} \lesssim y^{\perp} \wedge h^{\perp} .
$$

Proof. There exists $h \in \mathrm{C}(L)$ such that:

$$
\begin{gathered}
a \wedge b^{\perp} \wedge h \lesssim a^{\perp} \wedge b \wedge h, \\
a^{\perp} \wedge b \wedge h^{\perp} \lesssim a \wedge b^{\perp} \wedge h^{\perp} .
\end{gathered}
$$

(3) As $a \wedge\left(a^{\perp} \vee b\right) \backsim b \wedge\left(b^{\perp} \vee a\right), h \wedge a \wedge\left(a^{\perp} \vee b\right) \backsim h \wedge b \wedge\left(b^{\perp} \vee a\right)$ holds 
and

$$
\begin{aligned}
& a^{\perp} \wedge\left(a \vee b^{\perp}\right) \backsim b^{\perp} \wedge\left(b \vee a^{\perp}\right) \\
& \text { implies } h^{\perp} \wedge a^{\perp} \wedge\left(a \vee b^{\perp}\right) \backsim h^{\perp} \wedge b^{\perp} \wedge\left(b \vee a^{\perp}\right) .
\end{aligned}
$$

We have:

$$
\left[\left(a \wedge b^{\perp}\right) \wedge h\right] \vee\left[\left(a^{\perp} \wedge b\right) \wedge h\right] \leq\left(a \wedge b^{\perp}\right) \vee\left(a^{\perp} \wedge b\right)
$$

and

$$
\begin{aligned}
{\left[h \wedge a \wedge\left(a^{\perp} \vee b\right)\right] \vee\left[h \wedge b \wedge\left(b^{\perp} \vee a\right)\right] } & \leq\left[\left(a \wedge\left(a^{\perp} \vee b\right)\right] \vee\left[b \wedge\left(b^{\perp} \vee a\right)\right]\right. \\
& =(a \vee b) \wedge\left(b^{\perp} \vee a\right) \wedge\left(a^{\perp} \vee b\right) \\
& \leq\left(b^{\perp} \vee a\right) \wedge\left(a^{\perp} \vee b\right) \\
& =\left[\left(b \wedge a^{\perp}\right) \vee\left(a \wedge b^{\perp}\right)\right]^{\perp}
\end{aligned}
$$

By using (1), (3), and the additivity theorem for strong perspectivity, we have:

$$
\left(a \wedge b^{\perp} \wedge h\right) \vee\left[h \wedge a \wedge\left(a^{\perp} \vee b\right)\right] \lesssim\left(a^{\perp} \wedge b \wedge h\right) \vee\left[h \wedge b \wedge\left(b^{\perp} \vee a\right)\right]
$$

that is, $h \wedge a \lesssim h \wedge b$. Similarly, by using (2) and (4), we obtain $h^{\perp} \wedge a^{\perp} \lesssim$ $h^{\perp} \wedge b^{\perp}$.

Remark 1.

(a) The horizontal sum of $2^{3}$ (the Boolean lattice with three atoms) and $2^{2}$ satisfies (A.C.) ${ }^{\prime}$ but does not satisfy (A.C.). Hence, property (A.C.) $)^{\prime}$ is strictly weaker than (A.C.).

(b) Property (A.C.) ${ }^{\prime}$ is not satisfied by the horizontal sum of $2^{3}$ and $2^{4}$.

(c) In an irreducible OML $L$, (A.C.) and (A.C.)' become:

(A.C.): For all $a, b$ in $L, a \lesssim b$ or $b \lesssim a$,

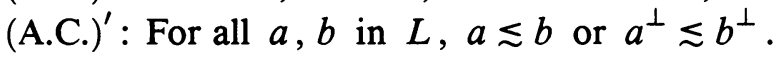

Hence, by analogy with a concept used in the dimension theory in complete OMLs, irreducible OMLs satisfying (A.C.) can be considered as the factors for the relation of strong perspectivity.

Proposition 4. The following statements on an orthomodular lattice $L$ are equivalent:

(a) L satisfies (A.C.).

(b) For all $x, y \in L, x \perp y$ implies that there exists a central element $h$ such that ( $\perp-A . C$.$) :$

$$
x \wedge h \lesssim y \wedge h \text { and } y \wedge h^{\perp} \lesssim x \wedge h^{\perp} \text {. }
$$

As the proof of Proposition 3, the proof of this result uses the parallelogram law for Sasaki projections and the theorem of additivity of strong perspectivity. Remark 2. In a Baer ${ }^{*}$-ring, the analogue of this proposition is false for equivalence of projections [2, $§ 14$, Exercise 1]. 


\subsection{The congruence lattice of an orthomodular lattice with (A.C.)}

Proposition 5. Let $I$ be an orthomodular ideal of an orthomodular lattice $L$ satisfying (A.C.)' Every central element of $L / I$ is the class modulo $I$ of a central element of $L$.

Proof. Let $[x]$ be the class of $x \in L$ in $L / I$ and remark that, for elements $x$ and $y$ of $L, x \sim y$ implies $[x] \sim[y]$. Let $[a] \in \mathrm{C}(L / I)$. There exists $h \in \mathrm{C}(L)$ such that $a \wedge h \lesssim a^{\perp} \wedge h$ and $a^{\perp} \wedge h^{\perp} \lesssim a \wedge h^{\perp}$. Hence, in $L / I$, we have $[a] \wedge[h] \lesssim[a]^{\perp} \wedge[h]$ and $[a]^{\perp} \wedge[h]^{\perp} \lesssim[a] \wedge[h]^{\perp}$. As these elements belong to the center of $L / I,[a] \wedge[h] \leq[a]^{\perp} \wedge[h]$ and $[a]^{\perp} \wedge[h]^{\perp} \leq[a] \wedge[h]^{\perp}$ holds. Therefore, $[a]=[h]^{\perp}$.

Corollary 1. Let $I$ be an orthomodular ideal of an orthomodular lattice $L$ satisfying (A.C.)'. The quotient $L / I$ is irreducible if and only if, for every central element $h$ of $L, h \in I$ or $h^{\perp} \in I$.

Writing out the proof would remind the reader of the definition of "irreducible."

Proposition 6. The congruence lattice of an irreducible orthomodular lattice with (A.C.) is a chain with a coatom.

Proof. Let $I$ and $J$ be two orthomodular ideals of an OML $L$ with (A.C.). If $I \subset J$ does not hold, then there exists $y \in I-J$. Let $x \in J$; as $y \lesssim x$ implies $y \in J, y \leqslant x$ does not hold. Hence, by Remark 1(c), $x \leqslant y$ and therefore $x \in I$ and $J \subset I$. The set of all orthomodular ideals of $L$, ordered by inclusion, is a chain and, by Zorn's lemma, this chain possesses a coatom.

Remark 3. (a) Let $H$ be a Hilbert space of infinite dimension $\aleph_{\alpha}$ and let $L$ be the OML of all closed subspaces of $H$. Since $L$ is complete and has the relative center property, $L$ satisfies (A.C.), and by [6] and [24], the orthomodular ideals of $L$, different from $\{0\}$, are the sets $J_{\beta}=\left\{X \in L \mid \operatorname{dim} X<\aleph_{\beta}\right\}$ for all ordinals $\beta \leq \alpha+1$. Hence, the lattice of orthomodular ideals of $L$ and the lattice of congruence relations of $L$ are well-ordered chains isomorphic to the ordinal $1+\alpha+2$; that is $\alpha+3$ if $\alpha$ is finite and $\alpha+2$ if $\alpha$ is infinite.

Since, for an OML $L$, a binary relation on $L$ is a congruence relation of the orthocomplemented lattice $L$ if and only if this relation is a tolerance of the lattice $L$ [21], the above example generalizes the main result of [5].

(b) Let $L$ be the OML of all closed subspaces of an infinite dimensional Hilbert space $H$. Consider an element $M$ of $L$ such that $M$ and $M^{\perp}$ are of infinite dimension, and let $I$ be the orthomodular ideal of all finite dimensional subspaces of $H$. If $[M]$ denotes the class of $M$ in $L / I$, then $L_{1}=I \cup I^{\perp} \cup$ $[M] \cup[M]^{\perp}$ is a subalgebra of $L$, since this subset is the inverse image of the subalgebra $\left\{0,1,[M],[M]^{\perp}\right\}$ of $L / I$ by the canonical homomorphism from $L$ onto $L / I$. Note that, for an element $a$ of $L_{1}$, the set of atoms which are lower bounds of $a$ is the same in $L$ and in $L_{1}$. This implies that $L_{1}$, as well as $L$, is a hyperirreducible OML (i.e., every interval $[0, x]$ is irreducible). In 
particular, $L_{1}$ is irreducible and satisfies the relative center property. Since $L_{1} / I$ is a four-element Boolean algebra, a consequence of Proposition 5 is that (A.C.) does not hold in $L_{1}$. This example, suggested by R. Greechie, shows that the relative center property does not imply (A.C.) for noncomplete OMLs.

(c) An OML with (A.C.) is irreducible if and only if it possesses solely one maximal orthomodular ideal. By Proposition 6 the condition is necessary, and for the converse, it suffices to remark that if $h$ is a central element different from 0 and 1 , then the maximal orthomodular ideals containing $[0, h]$ and $\left[0, h^{\perp}\right]$ are different.

Proposition 7. Let $L$ be an orthomodular lattice with (A.C.). The correspondence $I \rightarrow I \cap \mathrm{C}(L)$ pairs bijectively the maximal orthomodular ideals of $L$ with those of the center of $L$.

Proof. If $I$ is a maximal orthomodular ideal of $L$, then $I \cap \mathrm{C}(L)$ is an ideal of $\mathrm{C}(L)$ and $L / I$ is irreducible since $L / I$ is simple. By Corollary $1, I \cap \mathrm{C}(L)$ is a maximal ideal of the Boolean lattice $\mathrm{C}(L)$.

Let $I$ and $J$ be two orthomodular ideals of $L$, different from $L$, such that $I \cap \mathrm{C}(L)=J \cap \mathrm{C}(L)$. Assume that $J$ is maximal and that there exists $x \in I-J$. Let $y \in J$. As (A.C.) holds, there exists $h \in \mathrm{C}(L)$ satisfying $x \wedge h \lesssim y \wedge h$ and $y \wedge h^{\perp} \lesssim x \wedge h^{\perp}$. Since $x \wedge h^{\perp} \in I$, we have $y \wedge h^{\perp} \in I$ and $y \wedge h \in J$ implies $x \wedge h \in J$. As $x=(x \wedge h) \vee\left(x \wedge h^{\perp}\right) \notin J, x \wedge h^{\perp} \notin J$ holds. A fortiori, $h^{\perp} \notin J$; and, since $J \cap \mathrm{C}(L)$ is a maximal ideal of $\mathrm{C}(L)$, $h \in J$. By $I \cap \mathrm{C}(L)=J \cap \mathrm{C}(L), h \in I$ and therefore $y \wedge h \in I$. Finally, $y=(y \wedge h) \vee\left(y \wedge h^{\perp}\right) \in I$, and $J$ is a proper subset of $I$ contrary to the hypothesis that $J$ is maximal. Hence $I-J=\varnothing$ and $I \subset J$. Therefore, if $I$ is a maximal orthomodular ideal, $I \cap \mathrm{C}(L)=J \cap \mathrm{C}(L)$ implies $I=J$.

Now let $I$ be a maximal ideal of $\mathrm{C}(L)$ and let $I^{+}=\{x \in L \mid x \leq i$ for some $i$ in $I\}$. As $I^{+}$is a proper orthomodular ideal of $L$ there exists, by Zorn's Lemma, a maximal orthomodular ideal $J$ such that $I^{+} \subset J$. If $x \in$ $J \cap \mathrm{C}(L)-I^{+} \cap \mathrm{C}(L)$, then $x \notin I$. As $I$ is maximal, $x^{\perp} \in I$ and therefore $x^{\perp} \in I^{+} \cap \mathrm{C}(L)$. Since $I^{+} \cap \mathrm{C}(L) \subset J \cap \mathrm{C}(L), 1=x \vee x^{\perp} \in J \cap \mathrm{C}(L)$, a contradiction. Hence $J \cap \mathrm{C}(L)=I^{+} \cap \mathrm{C}(L)=I$, and the correspondence $I \rightarrow I \cap \mathrm{C}(L)$ is onto.

Remark 4. (a) Corollary 1 and Propositions 6 and 7 allow us to describe the top of the congruence lattice $\operatorname{Con}(L)$ of an OML $L$ with (A.C.). Let $\left(I_{i}\right)_{i \in I}$ be the family of maximal ideals of $\mathrm{C}(L)$, and let $I_{i}^{+}=\{x \in L \mid x \leq y$ for one $y \in I_{i}$, the orthomodular ideal of $L$ generated by $I_{i}$. The quotient $L / I_{i}^{+}$is, by Corollary 1 , irreducible and it satisfies (A.C.). Hence, the interval $\left[I_{i}^{+}, L\right]$ of $\operatorname{Con}(L)$ is isomorphic to the chain $\operatorname{Con}\left(L / I_{i}^{+}\right)$and the top of $\operatorname{Con}(L)$ is made up by chains linking together $L$ and the orthomodular ideals of $L$ generated by the maximal ideals of the center of $L$.

(b) If $A$ is a Baer ${ }^{*}$-ring which satisfies the condition that left projections are equivalent (in the sense of von Neumann) to right projections, then there exists 
a bijection between the set of all maximal ideals closed under equivalence of projections (S. K. Berberian calls them " $p$-ideals") and the set of all maximal ideals of the center $A[2, \S 24$, Proposition 3]. In such rings every $p$-ideal is an orthomodular ideal. It is apparently not known whether every orthomodular ideal is a $p$-ideal. It is for this reason that we prefer to call the kernels of congruence relations on OMLs orthomodular ideals rather than $p$-ideals.

(c) Proposition 4 is proved in [15] for continuous geometries, and there exists a similar result for operator algebras [2, §24, Exercise 4], [23]. Note that the proof of the proposition is based on the property of weak centrality of OMLs with (A.C.): If $I \cap \mathrm{C}(L)=J \cap \mathrm{C}(L)$ for two maximal orthomodular ideals $I$ and $J$, then $I=J$. This property has been introduced by Y. Misonou [19] for operator algebras.

\section{The CENTRAL COVER}

2.1. Condition (C). Recall that, in an OML $L$, the complements of an element $a$ are the elements $\varphi_{x}\left(a^{\perp}\right) \vee\left(x^{\perp} \wedge a^{\perp}\right)$ for arbitrary $x$ in $L$, and recall that two elements $a$ and $b$ are said to be in position $P^{\prime}$ if $a \wedge b^{\perp}=a^{\perp} \wedge b=0$.

Lemma 1. If $L$ is a complete or a commutator-finite orthomodular lattice, then for every $a$ in $L$ :

$$
\begin{aligned}
\bigvee\left\{x \mid x \text { a complement of } a^{\perp}\right\} & =\bigvee_{x \in L} \varphi_{x}(a) \\
& =\bigvee\left\{x \in L \mid P^{\prime}(x, a)\right\}=a \vee \bigvee_{x \in L} \operatorname{Com}(a, x) .
\end{aligned}
$$

Proof. If $L$ is a complete lattice:

$$
\bigvee\left\{x \mid x \text { is a complement of } a^{\perp}\right\}=\bigvee_{x \in L} \varphi_{x}(a) \vee\left(x^{\perp} \wedge a\right)=\bigvee_{x \in L} \varphi_{x}(a),
$$

and $x$ is a complement of $a^{\perp}$ means that $x$ and $a$ are in position $P^{\prime}$. Since $\varphi_{x}(a) \vee \varphi_{x}^{\perp}(a)=a \vee \operatorname{Com}(a, x)$, those elements equal $a \vee \bigvee_{x \in L} \operatorname{Com}(a, x)$ Now if $L$ is commutator-finite (see [11] for information about commutatorfinite OMLs), then $\left\{\varphi_{x}(a) \vee \varphi_{x^{\perp}}(a) \mid x \in L\right\}$ is finite and $\bigvee_{x \in L} \varphi_{x}(a)$ exists. Thus, the previous proof works.

We say that an OML $L$ satisfies Condition (C) if, for every $a \in L, \bigvee_{x \in L} \varphi_{x}(a)$ exists and is a central element. Proposition 10 will prove that a large class of complete OMLs satisfies this condition.

Proposition 8. If $L$ is an orthomodular lattice satisfying (C) then, for every element $a \in L$, the central cover of $a$ exists and $|a|=\bigvee_{x \in L} \varphi_{x}(a)$.

Proof. As $\varphi_{a}(a)=a, \bigvee_{x \in L} \varphi_{x}(a) \geq a$ holds. If $h \geq a$ is a central element, then $[0, h]$ is an orthomodular ideal and therefore, for all $x$ in $L, \varphi_{x}(a) \leq h$ so that $|a|=\bigvee_{x \in L} \varphi_{x}(a)$ since this join is central by hypothesis.

Recall a definition introduced in [6]. 
Definition 2. A binary relation $R$ on an orthomodular lattice $L$ characterizes the orthomodular ideals of $L$ if, for every ideal $I$ of $L$, the following statements are equivalent:

(a) The ideal $I$ is orthomodular.

(b) $x \in I$ and $R(x, y)$ imply $y \in I$.

It is well known that perspectivity, strong perspectivity, and position $P^{\prime}$ characterize orthomodular ideals in every OML.

Proposition 9. Let $R$ be a binary relation which characterizes orthomodular ideals of an orthomodular lattice $L$ satisfying (C). If $R$ contains position $P^{\prime}$ then:

$$
|a|=\bigvee\{x \in L \mid R(x, a)\} .
$$

In particular,

$$
|a|=\bigvee\{x \in L \mid x \text { perspective to } a\}=\bigvee\{x \in L \mid x \text { strongly perspective to } a\} \text {. }
$$

Proof. If $R$ characterizes orthomodular ideals, then $R(a, x)$ implies $x \leq|a|$. As $|a|=\bigvee_{x \in L}\left\{x \in L \mid P^{\prime}(a, x)\right\},|a|=\bigvee\{x \in L \mid R(x, a)\}$ holds if $R$ contains, as do perspectivity and strong perspectivity [6], position $P^{\prime}$.

Remark 5. (a) Let $R$ be the binary relation defined in an OML $L$ by $R(a, x) \Leftrightarrow$ $a^{\perp} \wedge x=0$. As this relation contains the position $P^{\prime}$ and characterizes orthomodular ideals, we have, if $L$ satisfies condition (C), $|a|=\bigvee\left\{x \in L \mid a^{\perp} \wedge x=0\right\}$ for all $a$ in $L$.

(b) If $a$ is an element of an OML $L$ satisfying condition (C) then:

$$
|a| \wedge\left|a^{\perp}\right|=\bigvee_{x \in L} \operatorname{Com}(a, x) \text {. }
$$

In particular, $\bigvee_{x \in L} \operatorname{Com}(a, x)$ is a central element, and $|a|=\bigvee_{x \in L} \operatorname{Com}(a, x)$ is equivalent to $\left|a^{\perp}\right|=1$.

(c) In the Dilworth lattice $D_{16}$, it is easy to find an element $a$ such that $|a| \neq \bigvee_{x \in L} \varphi_{x}(a)$. Hence, there exist complete OMLs which do not satisfy condition (C).

(d) For a complete OML $L$, condition (C) has an interpretation in $S(L)$, the Baer ${ }^{*}$-semigroup of all residuated mappings of $L$. Define:

$$
\gamma: a \in L \rightarrow|a| \text { and } f: a \in L \rightarrow \bigvee_{x \in L} \varphi_{x}(a) .
$$

It is easy to prove that $\gamma \in S(L)$ and, more precisely, that $\gamma$ is a projection of $S(L)$ (i.e., $\gamma=\gamma^{2}=\gamma^{*}$ ). We also have $f \in S(L)$ and $f=f^{*}$. One can prove that the following statements are equivalent:

(a) $L$ satisfies (C),

(b) $f$ is a projection, and

(c) $f=\gamma$. 


\subsection{The condition $(\mathrm{C})$ in complete orthomodular lattices}

Proposition 10. Let $L$ be a complete orthomodular lattice. The following statements are equivalent:

(a) For elements $x, y$ and $z$ of $L, y S^{1} z$ implies $y S^{1} \varphi_{x}(z)$,

(b) $\bigvee_{x \in L} \varphi_{x}(a)$ is a central element for all $a$ in $L$ (i.e., $L$ satisfies $(\mathrm{C})$ ),

(c) $|a|=\bigvee_{x \in L} \varphi_{x}(a)$ for all $a$ in $L$, and

(d) $S^{1}=S^{\infty}$.

Proof. (a) implies (b). As $x S^{1} y$ is equivalent to $y$ is less than or equal to all complements of $x$, Lemma 1 implies $\left(\bigvee_{x \in L} \varphi_{x}(a)\right)^{\perp} S^{1} a$. Hence, by using claim (a), $\left(\bigvee_{x \in L} \varphi_{x}(a)\right)^{\perp} S^{1} \varphi_{x}(a)$ holds for all $x$ in $L$. Therefore, $\left(\bigvee_{x \in L} \varphi_{x}(a)\right)^{\perp} S^{1} \bigvee_{x \in L} \varphi_{x}(a)$, and thus $\bigvee_{x \in L} \varphi_{x}(a)$ is a central element.

(b) implies (c). It is the above Proposition 8.

(c) implies (d). Since $S^{\infty} \subset S^{1}$ is always true it suffices to prove the reverse inclusion. Assume (c) and $a S^{1} b$ for elements $a$ and $b$ of $L$. By [13, $\S 8$, Exercise (with solution) 7], $\varphi_{x}(a) \perp b$ holds for all $x$ in $L$, and hence we have $|a|=\bigvee_{x \in L} \varphi_{x}(a) \perp b$. Therefore $|a| \perp|b|$, which is equivalent to $a S^{\infty} b$ in a complete OML [13, §8, Theorem 13]. For the proof of (d) implies (a) it is convenient to separate out a lemma.

Lemma 2. If $a$ and $b$ are two elements in position $P^{\prime}$ in an orthomodular lattice $L$, then $\varphi_{b}([0, a]) \subset[0, b]$ and, for every $x$ in $[0, a], x$ and $\varphi_{b}(x)$ are in position $P^{\prime}$.

Proof. As $a$ and $b$ are in position $P^{\prime}, \varphi_{b}(a)=b$, and since $\varphi_{b}$ is an isotone map, $\varphi_{b}([0, a]) \subset[0, b]$. Let $x \in[0, a]$. We have:

$$
\begin{gathered}
x \wedge\left(\varphi_{b}(x)\right)^{\perp}=x \wedge\left(b^{\perp} \vee\left(x^{\perp} \wedge b\right)\right)=\left(x \wedge b^{\perp}\right) \vee\left(x \wedge x^{\perp} \wedge b\right) \leq a \wedge b^{\perp}=0, \\
x^{\perp} \wedge\left(\varphi_{b}(x)\right)=x^{\perp} \wedge b \wedge\left(b^{\perp} \vee x\right)=\left(x \vee b^{\perp}\right)^{\perp} \wedge\left(b^{\perp} \vee x\right)=0 .
\end{gathered}
$$

(d) implies (a). Assume $y S^{1} z$ and let $y^{\prime} \leq y$ and $z^{\prime} \leq \varphi_{x}(z)$ such that $y^{\prime} \sim z^{\prime}$. As $\varphi_{x}(z)$ and $\varphi_{z}(x)$ are in position $P^{\prime}$, we infer, using Lemma 2 , that so are $z^{\prime}$ and $\varphi_{\varphi_{z}(x)}\left(z^{\prime}\right)$. By [6], $z^{\prime} \backsim \varphi_{\varphi_{z}(x)}\left(z^{\prime}\right)$ holds and, as $\varphi_{\varphi_{z}(x)}\left(z^{\prime}\right) \leq$ $\varphi_{z}(x) \leq z$, claim (d) implies $y^{\prime}=\varphi_{\varphi_{z}(x)}\left(z^{\prime}\right)=0$. Therefore, $y S^{1} \varphi_{x}(z)$ holds and (a) is proved.

Remark 6. (a) In the proof of (d) $\Rightarrow$ (a) we only use $S^{1}=S^{2}$, but it is known that $S^{n}=S^{n+1}$ is equivalent to $S^{n}=S^{\infty}[13, \S 8]$.

(b) The statement (a) of Proposition 10 is equivalent to $I_{a}^{*}=\left\{x \in L \mid a S^{1} x\right\}$ is, for all $a$ in $L$, an orthomodular ideal. One can prove that $I_{a}^{*}$ is, in the relatively pseudocomplemented lattice of all orthomodular ideals of $L$, the pseudocomplement of the orthomodular ideal $I_{a}$ generated by $a$. The ideal $I_{a}^{*}$ is a central element of the Mac Neille completion $\bar{L}^{+}$of $L$, and it is the orthocomplement of the central cover of the image of $a$ in $\bar{L}^{+}$. 


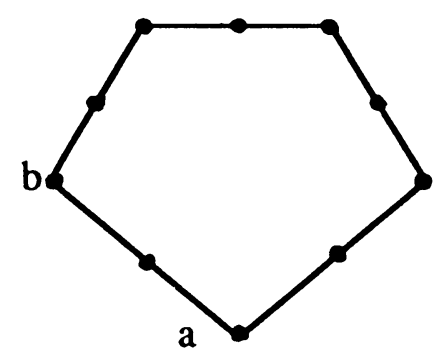

FIGURE 1

(c) Let $L$ be the OML with the Greechie diagram of Figure 1. In $L$, we have $S^{0} \neq S^{1}=S^{\infty}$ since $a S^{0} b$ holds and $x S^{1} y$ is never true if $x$ and $y$ belong to $L-\{0\}$.

Hence, condition (C) is strictly weaker than $S^{0}=S^{1}$, a condition equivalent to the relative center property in a complete OML [13, $\S 8$, Theorem 14].

2.3. Applications to Baer * ${ }^{*}$-rings and operator algebras. For a Baer ${ }^{*}$-ring $A$, $\operatorname{Proj}(A)$ denotes the complete orthomodular lattice of all projections of $A$.

Proposition 11. Let $A$ be a Baer ${ }^{*}$-ring satisfying the (SR)-axiom. For all $a \in$ $\operatorname{Proj}(A)$,

$$
\begin{aligned}
|a| & =\bigvee\{x \in \operatorname{Proj}(A) \mid x \text { equivalent to } a\} \\
& =\bigvee\{x \in \operatorname{Proj}(A) \mid x \text { unitary equivalent to } a\}
\end{aligned}
$$

Proof. Recall that in a Baer ${ }^{*}$-ring the (SR)-axiom implies the parallelogram law [17], and therefore equivalence of projections contains position $P^{\prime}[2, \S 13$, Proposition 4]. By [7], equivalence of projections characterizes the orthomodular ideals of $\operatorname{Proj}(A)$. Hence, since projection lattices of Baer * ${ }^{*}$-rings have the relative center property $[14$, Theorem 22], $\operatorname{Proj}(A)$ satisfies the condition (C) and Proposition 9 implies the first equality. For the second one, it suffices to use Proposition 9 and a result of [18]: two projections of a Baer * -ring satisfying the (SR)-axiom are unitary equivalent if and only if they are projective (projectivity is the transitive relation generated by perspectivity).

Remark 7. (a) Every $\mathrm{AW}^{*}$-algebra and, in particular, every von Neumann algebra satisfies the (SR)-axiom. The first equality of Proposition 11 is proved for $\mathrm{AW}^{*}$-algebras in [20].

(b) In [3] it is shown that in a Baer ${ }^{*}$-ring $A$ satisfying the parallelogram law and two other conditions denoted by $(*)$ and $(* *)$ and introduced in [18], the following statements are equivalent for all pairs of projections $a$ and $b$ :

(i) (a) and (b) are equivalent projections.

(ii) There exist orthogonal decompositions $a=a_{1} \vee a_{2}$ and $b=b_{1} \vee b_{2}$ where $a_{i}$ and $b_{i}$ are perspective. 
In such Baer *-rings, equivalence of projections contains position $P^{\prime}$ and characterizes the orthomodular ideals of $\operatorname{Proj}(A)$. Therefore, the first equality of Proposition 11 is also true for Baer ${ }^{*}$-rings satisfying the parallelogram law, $(*)$ and $(* *)$.

(c) In JW-algebras [22] equivalence of projections agrees with projectivity. Hence, as the orthomodular lattice of projections of a JW-algebra is complete and has the relative center property, the central cover is given by the first equality of Proposition 11.

\section{THE RELATIVE CENTER PROPERTY AND BAER *-SEMigrouPS}

Since the first definitions in the theory of Baer ${ }^{*}$-semigroups and Baer ${ }^{*}$-rings are incoherent, let us recall them.

A Baer ${ }^{*}$-semigroup (also known as a Foulis semigroup) $S$ is a ${ }^{*}$-semigroup such that, for each $a \in S, R(a)=\{x \in S \mid a x=0\}$ is a principal right ideal generated by a projection noted $a^{\prime}$. All such projections, called closed projections, form an OML denoted by $\operatorname{Proj}^{\prime}(S)$. When $\operatorname{Proj}^{\prime}(S)$ is a complete lattice, $S$ is said to be a complete Baer ${ }^{*}$-semigroup.

A *-ring in which the multiplicative *-semigroup is a Baer ${ }^{*}$-semigroup is called a Rickart ${ }^{*}$-ring, and a Rickart ${ }^{*}$-ring with a complete lattice of projections is a Baer ${ }^{*}$-ring. In a Rickart ${ }^{*}$-ring $A$, every projection is closed and $\operatorname{Proj}(A)$ denotes the OML of all projections of $A$.

We also recall that Baer ${ }^{*}$-semigroups and Rickart ${ }^{*}$-rings form equational classes of algebras [1], and therefore for these concepts the notion of subalgebra is unambiguous.

Some results of this section, such as Lemma 3, Propositions 13 and 14, are well known in the theory of Baer ${ }^{*}$-rings. In the case of Baer ${ }^{*}$-semigroups their proofs are necessary, since the additive structure is not available and, in particular, we do not have the important relation $p^{\prime}=1-p$ for a projection $p$.

3.1. Centers of a Baer ${ }^{*}$-semigroup. Let $S$ be a Baer ${ }^{*}$-semigroup. Define:

$$
\begin{aligned}
& \mathrm{Z}(S)=\{x \in S \mid x y=y x \text { for all } y \text { in } S\}, \\
& \mathrm{C}(S)=\left\{p \in \operatorname{Proj}^{\prime}(S) \mid p q=q p \text { for all } q \text { in } \operatorname{Proj}^{\prime}(S)\right\}
\end{aligned}
$$

Recall that $\mathrm{C}(S)$ is also the center of the orthomodular lattice $\operatorname{Proj}^{\prime}(S)$.

In general, $\mathrm{Z}(S) \cap \operatorname{Proj}^{\prime}(S) \subset \mathrm{C}(S)$, and the above example shows that strict inclusion is possible.

Example. Consider the Baer ${ }^{*}$-semigroup $S$ of all binary relations on a set $X$ introduced in [9]. Recall, for relations $R, R_{1}, R_{2}$, definitions of the three operations in $S$ :

$R_{1} R_{2}=\left\{(x, y) \mid\right.$ there exists $z \in X$ such that $(x, z) \in R_{1}$ and $\left.(z, y) \in R_{2}\right\}$,

$R^{*}=\{(x, y) \mid(y, x) \in R\}$,

$R^{\prime}=\{(x, x) \mid$ for all $y \in X,(y, x) \notin R\}$. 
The lattice $\operatorname{Proj}^{\prime}(S)$ is made up of all the identity relations on subsets of $X$ and is isomorphic to the Boolean lattice of subsets of $X$. Therefore, we have $\mathrm{C}(S)=\operatorname{Proj}^{\prime}(S)$.

Assume that $X$ has at least two elements and let $R$ be the identity relation on a nonempty subset $X_{0}$ of $X$, different from $X$. Consider $a \in X_{0}, b \notin X_{0}$, and $P=\{(a, b)\}$. As $R P=P$ and $P R=\varnothing, R P \neq P R$ holds and we have $\mathrm{Z}(S) \cap \operatorname{Proj}^{\prime}(S)=\{0,1\} \neq \mathrm{C}(S)$.

In the case of Rickart *-rings the situation is different and $\mathrm{Z}(S) \cap \operatorname{Proj}(S)=$ $\mathrm{C}(S)$ holds. This property is proved in a slightly different setting by S. Maeda in [16], and a proof is contained in an unpublished paper by S. K. Berberian [4]. For the convenience of the reader we give a proof of this important result.

Proposition 12. If $S$ is a Rickart ${ }^{*}$-ring then $\mathrm{Z}(S) \cap \operatorname{Proj}(S)=\mathrm{C}(S)$.

Proof. Let $e \in \mathrm{C}(S)$. As $e^{2}=e^{*}=e, e \in \mathrm{Z}(S)$ is equivalent to $e S e=e S$ and, by using $e^{\prime}=1-e$ in a Rickart *-ring, this last equality holds if and only if $e S e^{\prime}=0$.

Consider $g=e+e x e^{\prime}$. As $g^{2}=g, g(1-g)=0$ holds and therefore $g(1-g)^{* \prime \prime}=0$. Hence, $0=e(1-g)^{* \prime \prime}+e x e^{\prime}(1-g)^{* \prime \prime}=e(1-g)^{* \prime \prime}+$ ex $(1-g)^{* \prime \prime} e^{\prime}$. Right multiplication by $e$ gives $0=e(1-g)^{* \prime \prime} e=e(1-g)^{* \prime \prime}$. Hence $0=e(1-g)$, and $e=e g$ holds. As $e g=g$, we have $e=g$, which implies $e x e^{\prime}=0$, and $e S e^{\prime}=0$ is proved. Hence, we have $e \in Z(S)$.

Recall that by [9, Lemma 4] Z $(S)$ is, for every Baer ${ }^{*}$-semigroup $S$, a subalgebra of $S$. If $S$ is complete, then so is $\mathrm{Z}(S)$, and a subset $M$ of $\operatorname{Proj}^{\prime}(\mathrm{Z}(S))$ has the same infimum and supremum in $\operatorname{Proj}^{\prime}(S)$ and in $\operatorname{Proj}^{\prime}(Z(S))$.

3.2. The weak relative center property for complete Baer ${ }^{*}$-semigroups. We will prove that every complete Baer ${ }^{*}$-semigroup $S$ satisfies a property (see Proposition 15), called the weak relative center property, similar to the relative center property for OMLs where $\mathrm{Z}(e S e)$ replaces the center of the OML $[0, e]$ for all projections $e$.

Let $x$ be an element of a complete Baer ${ }^{*}$-semigroup $S$ and let $\gamma(x)$ be the infimum of $H=\left\{p \in \operatorname{Proj}^{\prime}(\mathrm{Z}(S)) \mid x=x p\right\}$. As $x=x p$ is equivalent to $p \geq x^{\prime \prime}, x^{\prime \prime}$ is a lower bound of $H$ in the lattice $\operatorname{Proj}^{\prime}(S)$. Since $H$ has the same infimum in $\operatorname{Proj}^{\prime}(S)$ and in $\operatorname{Proj}^{\prime}(Z(S))$, we have $\gamma(x) \geq x^{\prime \prime}$ and, therefore, $x=x \gamma(x)$. Note that if $S$ is a Baer "-ring and $p$ a projection, then by Proposition $12 \gamma(p)$ is the central cover of $p$ in the OML Proj $(S)$.

For the proof of the following proposition a lemma is useful.

Lemma 3. Let $I$ be a right ideal of a complete Baer ${ }^{*}$-semigroup $S$. The right annihilator of $I$ is a two-sided ideal generated by a projection of $\mathrm{Z}(S)$.

Proof. Since $S$ is complete, $R(I)=p S$ for a closed projection $p$. If $z \in R(I)$ and $y \in S$, then we have $(I y) z \subset I z=0$, and therefore $y z \in R(I)$, which is a two-sided ideal. Let $x \in S$. As $p \in p S, x p \in p S$ holds and $x p=p x p$. Similarly, we have $x^{*} p=p x^{*} p$, and therefore $p x=p x p$, which implies $p x=x p$ and so $p \in Z(S)$. 
Proposition 13. Let $S$ be a complete Baer ${ }^{*}$-semigroup. For all $x, y$ in $S$, $x S y=0$ is equivalent to $\gamma(x) \gamma(y)=0$.

Proof. Because $x=x \gamma(x), y=\gamma(y) y$, and $\gamma(y) \in \mathrm{Z}(S)$, we have $x S y=$ $x \gamma(x) \gamma(y) S y$ and so $\gamma(x) \gamma(y)=0$ implies $x S y=0$.

Now assume $x S y=0$. By Lemma 3, there exists $u \in \mathrm{Z}(S)$ such that $R(x S)=u S$. As $y \in R(x S), y=u y$ holds and $\gamma(y) \leq u$. By $u \in u S$, we obtain $x S u=0$ and so $x u=u x=0$. Hence, $x=u^{\prime} x$ holds and $\gamma(x) \leq u^{\prime}$. Thus, $\gamma(x) \gamma(y)=\left(\gamma(x) u^{\prime}\right)(u \gamma(y))=\gamma(x) u u^{\prime} \gamma(y)=0$.

Proposition 14 (The weak relative center property for complete Baer ${ }^{*}$-semigroups). Let $S$ be a complete Baer ${ }^{*}$-semigroup, $e \in \operatorname{Proj}^{\prime}(S)$, and $f \in$ $\operatorname{Proj}^{\prime}(e S e)$. The following statements are equivalent:

(a) $f \in \mathrm{Z}(e \mathrm{Se})$,

(b) $f=e \wedge \gamma(f)$.

Proof. Assume that $f=e \wedge \gamma(f)=e \gamma(f)$ and let exe $\in e S e$. We have fexe $=e \gamma(f) e x e=e \operatorname{exe}(f)=\operatorname{exee\gamma }(f)=\operatorname{exe} f$ and thus $f \in \mathrm{Z}(e S e)$.

Conversely, suppose that $f \in \mathrm{Z}(e S e)$ and let $x \in S$. By $f=e f=f e$, we obtain $f x f=f e x e f$, and as $f \in Z(e S e)$, fexe=exef holds. Hence, we have fexef=exef=exf, and thus $f x f=e x f$. This equality implies $f^{\prime} e x f=0$ and $f^{\prime} e S f=0$. By Proposition 13, we obtain $\gamma\left(f^{\prime} e\right) \gamma(f)=0$ and thus $f^{\prime} e \gamma(f)=0$. Hence, $e \gamma(f)=f^{\prime \prime} e \gamma(f)=f e \gamma(f)=f \gamma(f)=f$.

Remark 8. (a) The implication (a) $\Rightarrow$ (b) is proved in [14] for Baer *-rings. Actually, in [14], $\gamma(x)$ is denoted by $\mathrm{C}(x)$ and is the smallest central idempotent $p$ such that $x=p x$, but in a Rickart ${ }^{*}$-ring a central idempotent is a projection.

(b) Propositions 13 and 14 are proved for a weak Loomis * -semigroup in [11]. Recall that a weak Loomis ${ }^{*}$-semigroup is a complete Baer ${ }^{*}$-semigroup satisfying some additional properties verified in any multiplicative semigroup of a $\mathrm{W}^{*}$-algebra.

Definition 3. A Baer ${ }^{*}$-semigroup $S$ is said to be relatively central if, for all $e \in$ $\operatorname{Proj}^{\prime}(S), \mathrm{Z}(e S e) \cap \operatorname{Proj}^{\prime}(e S e)=\mathrm{C}(e S e)$. (This means that if a closed projection $p \leq e$ commutes with every closed projection $q \leq e$, then $p$ commutes with every element exe, $x \in S$.)

For a Rickart * -ring $A$, every subset $e A e$ for a projection $e$ is also a Rickart *-ring. Hence, by Proposition 12, every multiplicative semigroup of a Rickart *-ring is a relatively central Baer ${ }^{*}$-semigroup.

Proposition 15. A complete orthomodular lattice $L$ has the relative-center property if and only if it can be coordinatized by a relatively central Baer ${ }^{*}$-semigroup. Proof. By using Proposition 14, it is clear that a complete OML coordinatized by a relatively central Baer ${ }^{*}$-semigroup has the relative-center property. Conversely, consider an OML $L$ with the relative-center property and let $S$ be a Baer ${ }^{*}$-semigroup which coordinatizes $L$ and define $S_{0}=\left\{p_{1} \cdots p_{n} \mid p_{i} \in\right.$ $\left.\operatorname{Proj}^{\prime}(S)\right\}$. The set $S_{0}$ is a subalgebra of $S$ and $\operatorname{Proj}^{\prime}(S)=\operatorname{Proj}^{\prime}\left(S_{0}\right)$. Consider 
$e \in \operatorname{Proj}^{\prime}\left(S_{0}\right), f \in \mathrm{C}\left(e S_{0} e\right)$, and $h \in \mathrm{C}\left(\operatorname{Proj}^{\prime}\left(S_{0}\right)\right)$ such that $f=e \wedge h=e h$. For an element $e p_{1} \cdots p_{n} e$ of $e S_{0} e$ we have:

$$
f e p_{1} \cdots p_{n} e=e h e p_{1} \cdots p_{n} e=e^{2} p_{1} \cdots p_{n} e h=e p_{1} \cdots p_{n} e e h=e p_{1} \cdots p_{n} e f
$$

and, therefore, $f \in Z\left(e S_{0} e\right)$. Thus, $S_{0}$ is a relatively central Baer ${ }^{*}$-semigroup.

\section{REFERENCES}

1. D. H. Adams, Equational classes of Foulis semigroups and orthomodular lattices, Proc. Univ. Houston, Lattice Theory Conf., Houston, 1973.

2. S. K. Berberian, Baer ${ }^{*}$-rings, Springer-Verlag, Berlin, Heidelberg, and New York, 1972.

3. __ Equivalence and perspectivity in Baer rings, J. Algebra 87 (1984), 380-388.

4. __ Baer rings and Baer ${ }^{*}$-rings, 1988. (This paper is an unpublished English version of Anneaux et *-anneaux de Baer, Université de Poitiers (1982).)

5. I. Chajda and B. Zelinka, Every finite chain is the tolerance lattice of some lattice, Glas. Mat. Ser. III 20 (1985), 3-6.

6. G. Chevalier, Relations binaires et congruences dans un treillis orthomodulaire, C. R. Acad. Sci. Paris Sér. I Math. 299 (1984), 785-788.

7. __ Les congruences d'un treillis orthomodulaire de projections, C. R. Acad. Sci. Paris Sér. I Math. 299 (1984), 731-739.

8. _ Commutators and decompositions of orthomodular lattices, Order 6 (1989), 181-194.

9. D. J. Foulis, Relative inverses in Baer ${ }^{*}$-semigroups, Michigan Math. J. 10 (1963), 65-84.

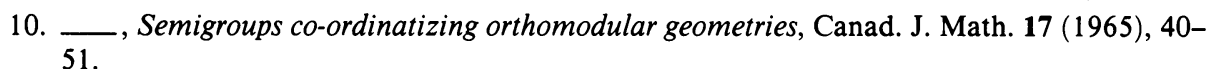

11. R. Greechie and L. Herman, Commutators-finite orthomodular lattices, Order 1 (1985), 277-284.

12. M. F. Janowitz, Separation conditions in relatively complemented lattices, Colloq. Math. 22 (1970), 25-34.

13. G. Kalmbach, Orthomodular lattices, Academic Press, London, 1983.

14. I. Kaplansky, Rings of operators, W. A. Benjamin, New York, 1968.

15. Y. Kawada, K. Higuti, und Y. Matusima, Bemerkungen zur vorangehenden Arbeit von Herrn T. Iwamura, Japan. J. Math. 19 (1944), 73-79.

16. S. Maeda, On the lattice of projections of a Baer ${ }^{*}$-ring, J. Sci. Hiroshima Univ. Ser. A 22 (1958), 75-88.

17. __ On ${ }^{*}$-rings satisfying the square root axiom, Proc. Amer. Math. Soc. (1975), 188-190.

18. S. Maeda and S. S. Holland, Jr., Equivalence of projections in Baer ${ }^{*}$-rings, J. Algebra 39 (1976), 150-159.

19. Y. Misonou, On a weakly central operator algebra, Tôhoku Math. J. (2) 4 (1952), 194-202.

20. U. Sasaki, Lattices of projections in $\mathrm{AW}^{*}$-algebras, J. Sci. Hiroshima Univ. Ser. A 19 (1955), $1-30$.

21. D. Schweigert, Compatible relations of modular and orthomodular lattices, Proc. Amer. Math. Soc. 81 (1981), 462-464.

22. D. Topping, Jordan algebras of self adjoint operators, Mem. Amer. Math. Soc., no. 53, Amer. Math. Soc., Providence, RI, 1965.

23. F. B. Wright, A reduction for algebras of finite type, Ann. of Math. (2) 60 (1954), 560-570.

24. __ The ideals in a factor, Ann. of Math. (2) 68 (1958), 475-483.

Université Lyon 1, Institut de Mathématiques et INFormatique de 1'ISM, 43 BD DU 11-Novembre-1918, 69622 Villeurbanne CedeX, France 